\title{
Contents, Vol. 107, 1944
}

\section{Index}

Amsler, M., A propos de la dacryo-cysto-rhinostomie . . $50-\cdot \mathrm{L} \cdot$ es divers elements des syndromes hypertensifs . . 79 Discussion: Wei'denberg, Goldmann.

- $\quad$ Destruction hypotonisante du corps ciliaire par diather-mocoagulation non perforante 95

Babel, J., L'action de la vitamine E sur la reunite pigmen-

taire expérimentale du lapin 102

Discussion: Franceschetti.

- vide Franceschetti, A.

Bangerter, A., Beiträge zur Diagnostik und Therapie des

Tränens 51

Diskussion: Patry, Goldmann.

- vide Goldmann, H.

Birkhäuser, R., Augenkrankheiten durch infektiöse Zahn-

herde 3

Diskussion: Dufour, Verrey.

Bíró, I., Ueber den Zusammenhang von Degeneratio pig-

mentosa retinae und Störungen des Gehörs . . . 149

Bischler, V., et Pictet, A., Malformations oculaires chez le

cobaye 116

Bruckner, A., Kurze Mitteilungen

Doppelseitiges Gliom, Röntgenbestrahlung eines Auges 28

Akkommodationslähmung nach Diphtherieschutz-impfung 29

Diskussion: Knapp, Rintelen.

Dufour, R.; vide Verrey, F.

Franceschetti, A., Le syndrome de Behr, ses rapports avec

la maladie de Leber et les hérédoataxies . . . 17

- Prothèses palpébro-oculaires exécutées d'après la technique des pieces anatomiques simili-vivantes du Prof.

de Seigneux 25

- $\quad$ et Babel, J., Essai de classification anatomique des dé-générescences familiales et héréditaires de la cornée 66

Goldmann, H., Ein neues Prinzip direkt registrierender

Adaptometer 55

Höhlenplastik 60

und Bangerter, A., Demonstrationen

Praktisches Adaptometer für den tägl. Gebrauch 75 
Lokalisation nichtmagnetischer oder auch magne-tischer kleinster wandständiger Fremdkörper . . 75

Lochfinder für die Operation der Netzhautablösung 76

Kammerwinkelkontaktglas 77

Funduskontaktglas 78

Methodik zur transvitralen Operation von Macula-löchern bei Netzhautablösung an der

Spaltlampe

mit Kontaktglas 78

Göthlín, G. F., Hemmungsprozesse in dem normalen Far-

bensinnmechanismus 158

Gruber, M., Histologische Augenhintergrundsbefunde bei

Fahrscher maligner Nephrosklerose 87

Klaingutí, R., Ueber Gewebsdisposition bei Ulcus trauma-

ticum simplex der Hornhaut 73

Knapp, P., Zur Therapie des Blepharoklonus mit Alkohol-

injektionen 64

Diskussion: Gol·dmann, Rintelen, Franceschetti.

Kuyz, O., Zur Frage der Netzhautzysten ... 233

Manen, J. G. van, Décollement rétinien falciforme congenital et anomalies congénitales

connexes .... 121

Pictet, A., vide Bischler, V.

Racine, W., Le larmoiement d'origine nasale... 42

Diskussion: Gol·dmann, Knapp, Franceschetti, Rintelen.

Radnót, M., Männliche Gonaden und intraokularer Druck 282

Rintelen, F., Die Therapie des Tränens 31

Streiff, E. B., Affections maculaires associées à des cavités

ou fossettes de la papille. (Avec planche III) ... 90 Verrey, F., et Dufour, R., Quelques aspects

cytologiques de

Thumeur aqueuse dans les uvéites 98

Discussion: Bruckner.

Wagner, H., Fundusveränderungen bei Faiirscher Nephrosklerose

Weber, E., Der vordere Glaskörperabschluß. Die spontane Linsenblattablösung im Senium - eine neue Spalt-lampendiagnose. (Hierzu Tafel I und II) . . . . 108

Weekers, R., Les cataractes par hypocalcémie et leur traite-

ment médicamenteux 257

UEBERSIGHTSREFERATE - REVIEWS - REVUES

Bischler, V., Rétine. (Années 1939, 1940, 1941) ... 290 Bruckner, A., Physiologische und psychologische Optik.

Berichtszeit 1939 bis $1942 \quad 178,310$

GESELLSCHAFTSBERICHTE - SOCIETY TRANSACTIONS - SOCIÉTÉS

Schweize IÍsche Ophthalmologische Gcsellschaft - Société SuissedOphtalmologie. 36.

Generalversammlung in Langenthal, 11. und12. September 1943 -36e Assemblée generate à

Langenthal, 11 et12 septembre 1943

$\mathrm{J}$

Sitzungsbericht der 107. Versammlung der Niederländischen Ophthal-

m. mologischen Gesellschaft in Utrecht am 18./19. Dezember 1943336 
BUCHBESPRECHUNGEN - BOOKS REVIEW - LIVRES NOUVEAUX 120Varia 120 